\title{
Technè
}

La science au service de l'histoire de l'art et de la préservation des biens culturels

41 | 2015

Arts textiles antiques et modernes. Approche scientifique et restauration

\section{La ville d'Akrotiri : une source exceptionnelle d'information sur l'activité textile au milieu du second millénaire au cœur du monde égéen}

The city of Akrotiri : an exceptional source of information on textile activity in the heart of the Aegean world in the mid-2nd millennium $B C$

\section{Stella Spantidaki et Christophe Moulherat}

\section{OpenEdition Journals}

Édition électronique

URL : http://journals.openedition.org/techne/4168

DOI : 10.4000/techne.4168

ISSN : 2534-5168

\section{Éditeur}

C2RMF

\section{Édition imprimée}

Date de publication : 25 novembre 2015

Pagination : 19-24

ISBN : 978-2-7118-6248-1

ISSN : $1254-7867$

\section{Référence électronique}

Stella Spantidaki et Christophe Moulherat, "La ville d'Akrotiri : une source exceptionnelle d'information sur l'activité textile au milieu du second millénaire au cœur du monde égéen », Technè [En ligne], 41 | 2015, mis en ligne le 08 août 2020, consulté le 11 mars 2021. URL : http://journals.openedition.org/ techne/4168; DOI : https://doi.org/10.4000/techne.4168

\section{(c)}

La revue Technè. La science au service de l'histoire de l'art et de la préservation des biens culturels est mise à disposition selon les termes de la Licence Creative Commons Attribution - Pas d'Utilisation Commerciale - Pas de Modification 4.0 International. 
Stella Spantidaki

Christophe Moulherat

\section{La ville d'Akrotiri : une source exceptionnelle d'information sur l'activité textile au milieu du second millénaire au cour du monde égéen}

The city of Akrotiri: an exceptional source of information on textile activity in the heart of the Aegean world in the mid-2nd millennium BC
Résumé. Akrotiri, située sur l'île de Santorin, est une ville exceptionnellement bien conservée grâce à une éruption volcanique qui s'est produite au milieu du XVII ${ }^{e}$ siècle avant notre ère. Elle a livré une grande variété d'objets du quotidien, dont plusieurs liés à la production des textiles, témoins d'une activité florissante. Nos sources d'information sont multiples, qu'il s'agisse de tablettes rédigées en Linéaire A relatives à la production et aux échanges de tissus, ou de peintures murales sur lesquelles est notamment représentée une grande diversité de vêtements. Les outils de filage et surtout la concentration de pesons de tissage témoignent d'une organisation de la production dans des espaces spécifiques.

Les tissus conservés ont été réalisés avec des matériaux d'origine végétale et animale et présentent parfois des traces de décoration. La découverte de feutre végétal, de filets de pêche, d'un cocon de soie et d'une grande variété de ficelles et cordes employées sous différentes formes livrent des informations sur la diversité des activités liées au travail des matières organiques et en particulier des textiles, confirmant ainsi la réalité des représentations que nous livrent les peintures murales.

Mots-clés. Akrotiri, Santorin, Théra, tissus, production
Abstract. Akrotiri, on the Greek island of Santorini, was a settlement buried in volcanic ash, and thus exceptionally wellpreserved, after an eruption in the mid-17th century $B C$. Excavations on the site have uncovered a wide variety of everyday objects, several of which are related to textile production and attest to a thriving industry. Our multiple sources of information include tablets bearing inscriptions in Linear A concerning textile production and trade, as well as mural paintings notably depicting garments in very diversified fabrics. Weaving tools and the abundance of loom weights testify to organized production in specific locations. The fabrics preserved were made from plant and animal fibres and sometimes show traces of decorative motifs. The discovery of plant fibre felt, fishing nets, a silk cocoon and a wide variety of strings and cords used in different manners has provided information about the diversity of activities related to working with organic materials, especially textiles, thus confirming the authenticity of what is represented in the murals.

Keywords. Akrotiri, Santorini, Thera, fabrics, textile production. textile.

\section{Contexte de la ville}

L'île de Santorin est située en mer Égée, au sud des Cyclades et au nord de la Crète, dans une zone de contact entre la Crète, l'Égypte, le Levant et la Grèce continentale. La recherche archéologique, initiée en 1967 par Spyridon Marinatos et poursuivie par Christos Doumas sous l'égide de la Société archéologique grecque, a mis au jour l'emplacement d'une ville détruite dans la deuxième moitié du XVII ${ }^{\mathrm{e}}$ siècle avant notre ère par l'éruption du volcan. Aujourd'hui, l'île est formée par les bords du cratère. Le site a été recouvert par des couches de cendres volcaniques qui ont préservé les structures architecturales et un très grand nombre d'objets du quotidien. Considéré comme «le Pompéi préhistorique », le site d'Akrotiri a révélé plusieurs types de matériaux et outils dans un état de conservation exceptionnel. L'éruption du volcan nous livre ainsi un instantané de la vie quotidienne des derniers habitants de la ville.

\section{Témoignages liés à l'activité textile}

Une des caractéristiques de la ville d'Akrotiri est qu'un grand nombre de peintures murales qui décoraient plusieurs bâtiments (fig. 1) y a été découvert. Ces images représentent un environnement imaginaire ou réel, des animaux, des scènes de la vie quotidienne et des scènes à caractère religieux. Un important projet de restauration a permis la reconstitution

Stella Spantidaki, Honorary Research Associate, UCL, Institute of Archaeology ; Centre Hellénique de Recherche et de Restauration de tissus archéologiques (ARTEX), Athènes (sspantidaki@yahoo.com). Christophe Moulherat, chargé d'analyses des collections/référent textile, musée du quai Branly (christophe.moulherat@quaibranly.fr). 


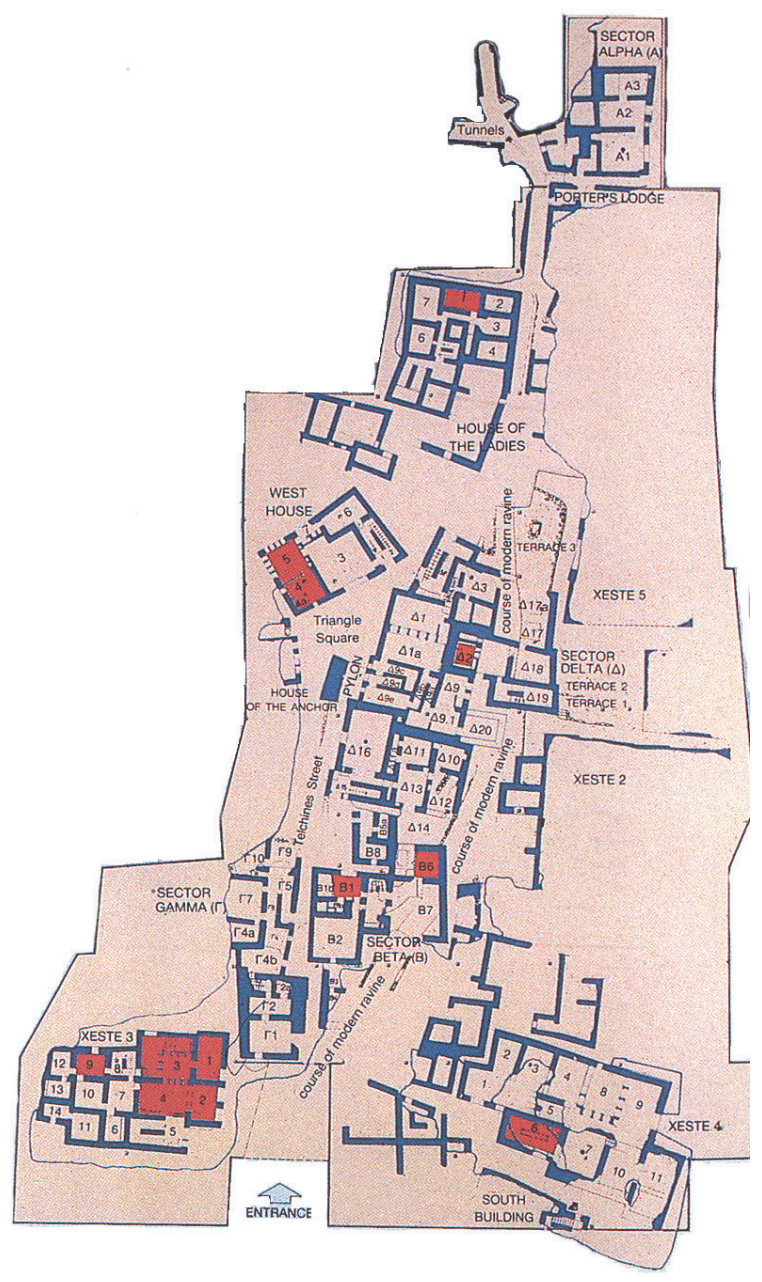

Fig. 1. Plan des fouilles de la ville d'Akrotiri. En rouge, les endroits où les peintures murales ont été découvertes. D'après Doumas.

de plusieurs peintures murales et la création de copies visibles au musée archéologique de Santorin et au Musée national d'Athènes, tandis que les pièces originales sont conservées dans les réserves de l'atelier de restauration d'Akrotiri ${ }^{1}$. Ces peintures murales représentent une source d'information précieuse sur les modes vestimentaires de l'époque. Le décor et les modes d'assemblage représentés ont fait l'objet de plusieurs études spécialisées ${ }^{2}$. Les peintures nous renseignent également sur d'autres produits textiles utilitaires rarement évoqués, comme des voiles de bateaux, des coussins, mais également sur l'importance et la diversité des cordes, cordelettes et tresses pour la réalisation de décor, de lit, etc. Un groupe de tablettes en Linéaire A découvert à Akrotiri nous livre des informations administratives sur la production textile de la ville. Une tablette contient l'idéogramme correspondant au mot « tissu » avec un chiffre indiquant plus de 200 pièces. C'est là, de toute évidence, l'enregistrement d'une énorme quantité de tissus, relative à un surplus ou à une opération commerciale ${ }^{3}$.

\section{Les objets liés au filage}

Parmi les objets qui prouvent une activité textile, on trouve des fusaïoles, témoignant d'une activité de filage au fuseau, et des pesons, qui nous informent sur la présence de métiers verticaux à poids, attestés en Grèce de l'âge du Bronze jusqu'à l'Antiquité tardive.

Un nombre très élevé de pesons de forme discoïde en terre cuite, très uniformes par leur forme et poids, a été découvert, portant une ou, plus rarement, deux perforations sur leur partie supérieure ${ }^{4}$. Ces pesons ont été mis au jour dans seulement quatre des onze maisons bien fouillées de la ville, prouvant ainsi qu'il n’y avait pas de métier à tisser vertical dans toutes les maisons. La plus grande concentration, 450 pesons environ, provient de la chambre 3 de la Maison Ouest, ce qui a permis de suggérer qu'il s'agissait d'un atelier de tissage, puisque ce dernier se faisait dans des lieux spécifiques ${ }^{5}$. Cette chambre, située au premier étage, est en effet munie de grandes fenêtres indispensables aux opérations de tissage ${ }^{6}$. Les outils de filage, les fusaïoles conservées, qu'elles soient en terre cuite ou en pierre, ont des formes différentes ${ }^{7}$, mais en nombre beaucoup plus réduit que les pesons. Ainsi, dans la Maison Ouest, sur 450 pesons exhumés, seules sept fusaioles ont été retrouvées ${ }^{8}$. Cependant, un petit nombre de fusaïoles mis au jour dans des contextes secondaires indique que cet outil pouvait circuler dans la ville ${ }^{9}$. Cette différence numérique soulève des questions concernant la technologie du filage (les fuseaux étaient-ils tous munis de fusaïoles ?) et le lieu de transformation des fibres en fil ${ }^{10}$. Tout laisse à croire que la production massive de fusaïoles et la fabrication du fil s'effectuaient à l'extérieur du centre urbain d'Akrotiri ${ }^{11}$. L'usage des pesons et les types de tissus qui pouvaient être produits avec ces outils ont été étudiés suivant une méthode élaborée au Centre for Textile Research de l'université de Copenhague ${ }^{12}$. Cette étude a été réalisée sur la base d'un nombre restreint de pesons trouvés en groupe dans le complexe D d'Akrotiri et elle montre qu'ils pouvaient être employés pour fabriquer des tissus de type différent ${ }^{13}$.

\section{Les matières végétales}

Les habitants d'Akrotiri savaient tirer profit des matières végétales, à partir desquelles ils réalisaient des tresses, des cordes de différentes épaisseurs, un grand nombre d'objets en vannerie ${ }^{14}$ et des filets de pêche. Autre témoignage de la parfaite maîtrise du monde végétal par les populations locales : la découverte de deux couvertures en feutre végétal disposées sur des pithoï. L'identification des matériaux amorcée par Christophe Moulherat est en cours d'achèvement par Lefteris Zorzos, de l'Institute of Archaeology de l'University College London à partir de l'analyse des phytolites ${ }^{15}$.

Fils et cordes constituent les preuves de l'activité de filage. Isolés ou groupés pour former des objets de structure plus complexe, ils sont des témoignages de la vie quotidienne. Deux groupes de fibres participent à la réalisation des fils, 


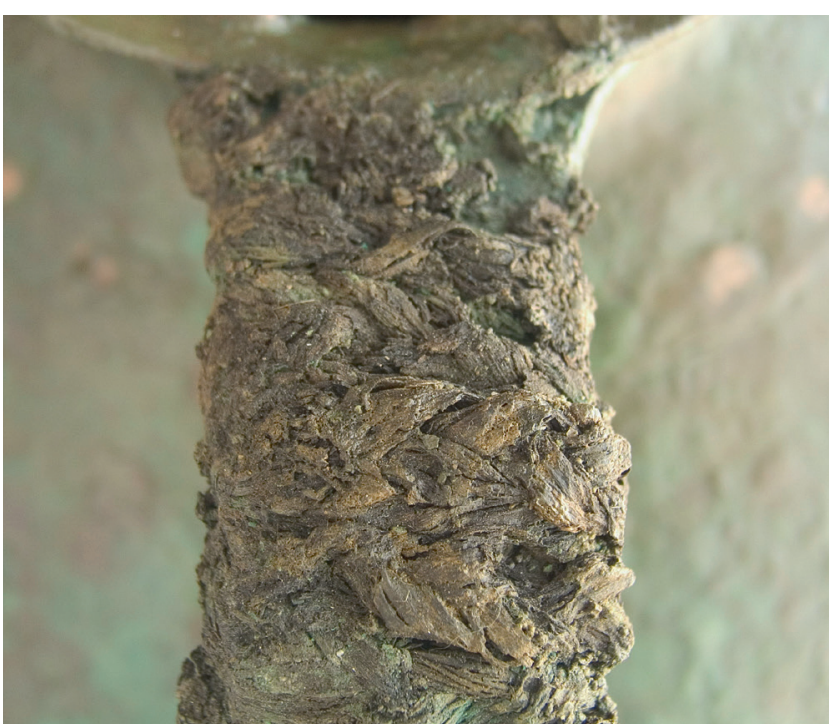

Fig. 2. Détail de la tresse végétale sur le vase métallique, pronchous, $\mathrm{n}^{\circ}$ d'inv. : 9214. (C) ARTEX/Christophe Moulherat.

les fibres végétales et les fibres animales. Les études montrent déjà la présence de laine, de lin et d'autres fibres libériennes non encore identifiées. Le nombre de brins impliqués diffère en fonction de l'utilisation souhaitée. Ils sont employés comme moyen de suspension pour des petits plateaux de balance ${ }^{16}$, figurés sur les peintures murales, où leur empreinte dessine et décore des lignes, mais ils forment aussi les sommiers de lits et des filets de pêche ${ }^{17}$. À Akrotiri, deux exemples de filets noués sont conservés. Les fibres ont une origine végétale libérienne et les fils sont retors de deux bouts ${ }^{18}$ (z2S) (fig. 3). Des tresses ont également été confectionnées pour isoler les anses de grandes jarres métalliques. On observe ce type d'aménagement sur les peintures murales ${ }^{19}$ (fig. 2).

\section{Les matériaux d'origine animale}

Les habitants d'Akrotiri se servaient également de matériaux d'origine animale : la découverte de fils de laine très fins provenant d'une toile représente l'un des plus anciens témoignages de l'emploi de la laine en Grèce ${ }^{20}$. Un cocon de soie sauvage a également été mis au jour à l'intérieur de la Maison Ouest $^{21}$; cette trouvaille pose la question de l'utilisation de la soie sauvage à des fins textiles à l'âge du bronze en Grèce et d'une façon générale dans le monde méditerranéen.

Enfin, des milliers de coquillages à pourpre communément appelés murex ont été retrouvés. Nous savons que la pourpre a été exploitée sous forme de pigment pour rehausser certains décors muraux, mais rien n'indique qu'elle ait ici pu être aussi utilisée en tant que colorant textile ${ }^{22}$. Pour le moment, en effet, il n'y a pas de traces de teinture à la pourpre sur les tissus découverts, ni celles d'autres couleurs également. Plusieurs raisons peuvent être ici invoquées : d'une part, l'état général des restes de tissu conservés le plus souvent sous une

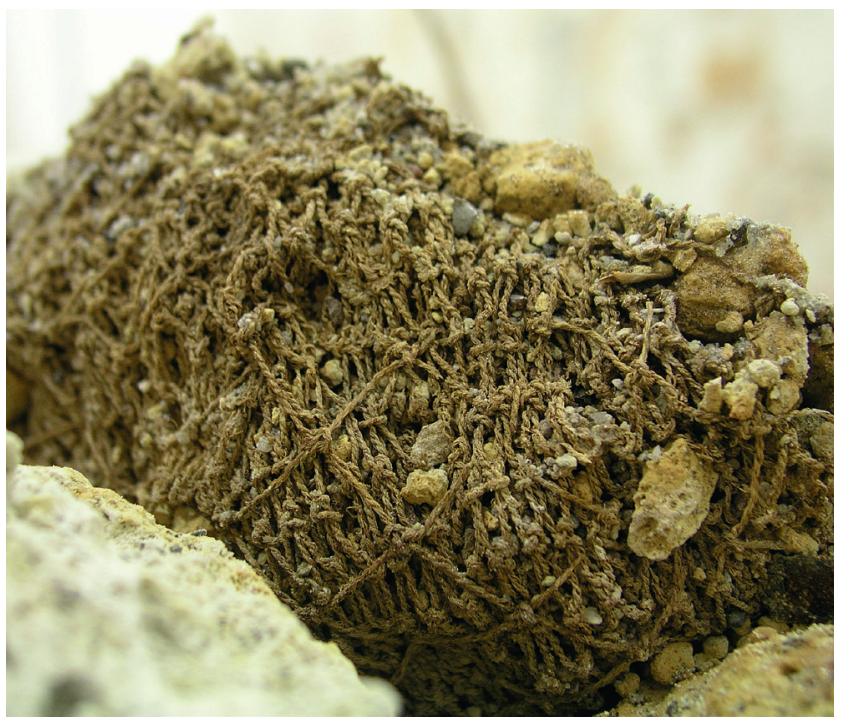

Fig. 3. Vue des filets de pêche. (๑) ARTEX/Christophe Moulherat.

forme carbonisée, d'autre part, la taille réduite (entre 1 et $10 \mathrm{~cm}$ ) et le peu de vestiges existants.

\section{Les vestiges textiles}

Les vestiges textiles trouvés à Akrotiri sont conservés soit sous forme minéralisée au contact avec des objets métalliques, soit isolés en de minuscules fragments carbonisés. Ces données archéologiques correspondent aux représentations murales et constituent la preuve d'une activité textile développée et variée. Le centre ARTEX ${ }^{23}$ a lancé l'étude des vestiges textiles d'Akrotiri en 2004.

En 1999, l'archéologue Giorgos Alexopoulos, lors de la fouille du pilier 52 du nouveau canopée, a découvert un tas d'ossements d'ovicapridés et la seule statuette en or d'Akrotiri représentant un bouc ${ }^{24}$. Vingt-cinq fragments du même tissu ont été récupérés parmi les ossements, mesurant de 0,5 à $3 \mathrm{~cm}$, avec une moyenne de $1,5 \mathrm{~cm}$. Ils ont été conservés grâce à la chaleur de l'éruption volcanique, qui, à ces conditions anaérobiques, a provoqué leur carbonisation. Il s'agit d'une toile équilibrée, avec des fils z2S et une réduction de 20-22 fils au centimètre dans les deux directions. Le diamètre des fils se situe entre 0,25 et $0,4 \mathrm{~mm}$, avec une moyenne de $0,3 \mathrm{~mm}$. Bien que les fils aient probablement rétréci lors de la carbonisation, ces diamètres indiquent un tissu très fin. L'identification des fibres a montré que les fils étaient en lin dans les deux directions (fig. 4).

Ce tissu conserve des traces de décorations variées :

- ourlet décoratif réalisé pour éviter que le tissu ne s'effile, et qui se distingue par le fait qu'il est rendu visible, notamment par un fil d'une autre couleur que le tissu.

- traces de broderie comme l'atteste la présence de fils de diamètre et de couleur différents introduits dans le tissu après tissage. 


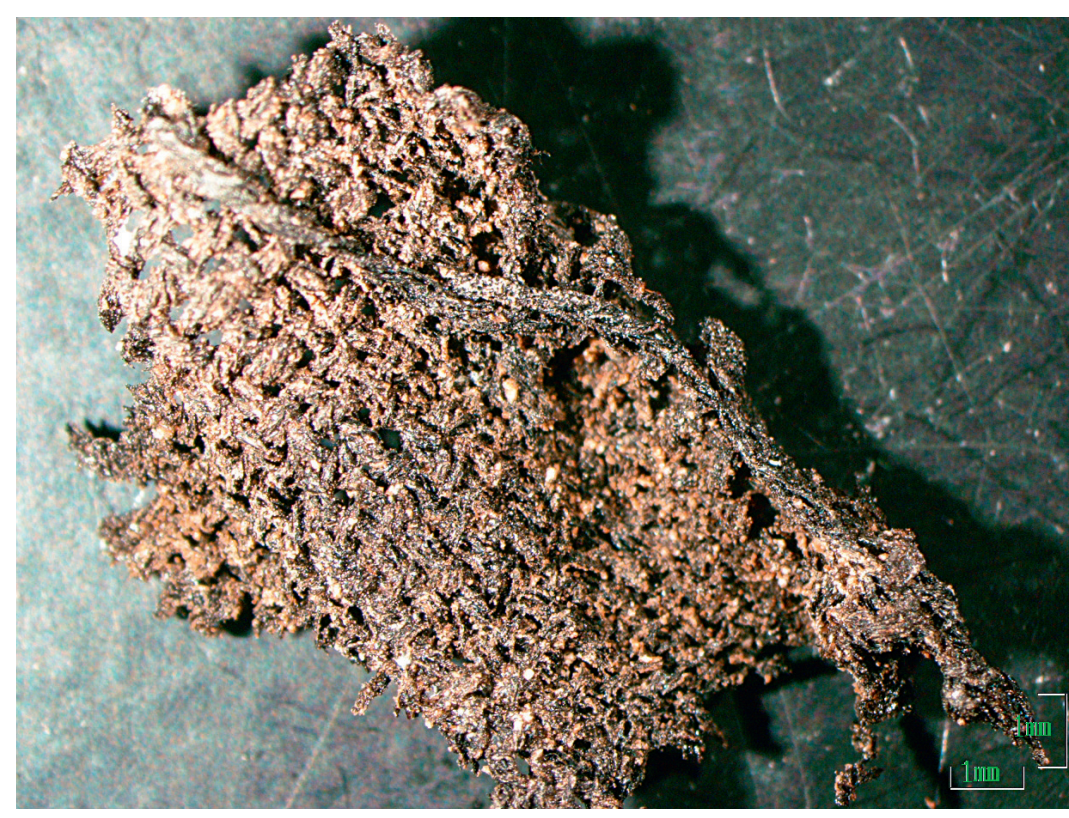

Fig. 4. Vue du tissu découvert au pilier 52n. (C) ARTEX/Christophe Moulherat.

- plusieurs types de franges qui se distinguent les uns des autres par leur taille et par la présence ou l'absence de petits nœuds.

En 2000, à l'endroit du puits 1b, ont été découverts des restes de tissu à côté de graines d'orge carbonisées. Ce voisinage a conduit à l'hypothèse d'un «sac » contenant ces graines. Trois plages avec des traces d'un tissu et de deux filets ont été mises en évidence. Le tissu est une toile équilibrée, dont le diamètre des fils diffère selon leur direction. Les fils de chaîne identifiés par une plus forte tension sont z2S, d'un diamètre entre 0,3 et $0,4 \mathrm{~mm}$ et avec une réduction de 5-6 fils

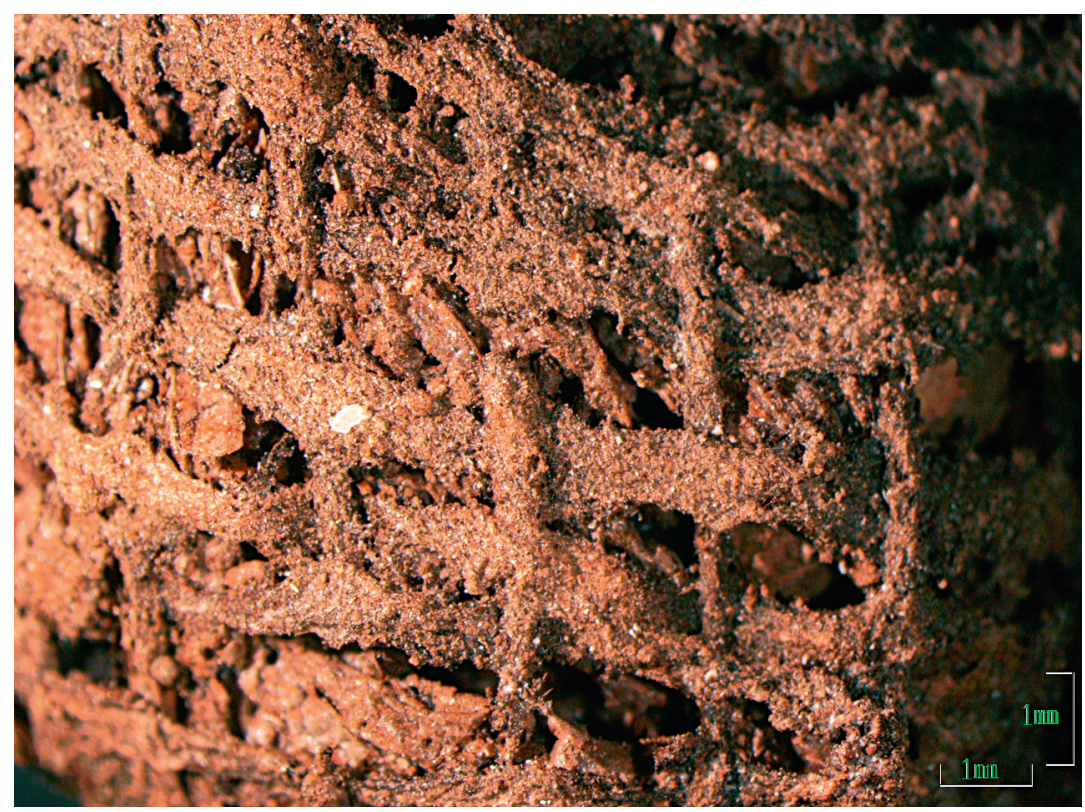

Fig. 5. Détail du sac à grains à la loupe binoculaire. (C) ARTEX/Christophe Moulherat. par cm ; au contraire, les fils de trame ont une réduction de 7 fils par $\mathrm{cm}$, avec un diamètre qui varie entre 0,6 et $2 \mathrm{~mm}$ sans torsion apparente. Ces fils qui ont l'aspect de lanières n’ont pas toujours la même largeur et ont été utilisés pour créer un décor de bandes ${ }^{25}$ (fig. 5). En 2000, des fragments d'un autre tissu ont été découverts à l'endroit du pilier 68a ; ils ont été identifiés à l'intérieur d'une couche de cendres volcaniques, dans un espace externe voisin d'une maison, parmi plusieurs produits de vannerie. Il s'agit d'une toile équilibrée mesurant $13 \times 8 \mathrm{~cm}$, disposée sous la forme de deux couches de tissus superposés. Aux endroits où l'on distingue la couche inférieure, mieux protégée, le tissu a gardé son aspect d'origine avec des fils plus gonflants. La zone centrale est plus altérée et l'impression générale d'un tissage aéré provient de cette altération. Les fils de deux directions n'ont pas les mêmes caractéristiques. Les fils de chaîne sont z2S, avec un diamètre de $0,70 \mathrm{~mm}$ et une réduction de 5 fils par $\mathrm{cm}$. Les fils de trame sont simples d'une nette torsion $\mathrm{z}$ avec un diamètre de $1,2 \mathrm{~mm}$ et une réduction de 5 fils par $\mathrm{cm}$. L'identification des fibres a montré qu'il s'agit de fibres libériennes dans les deux directions. Ce tissu constitue l'un des rares exemples en Grèce de toile présentant une structure de fil différente en chaîne et en trame. Cependant, si la chaîne a été identifiée comme du lin, les fils utilisés pour la trame, bien qu'appartenant également au règne végétal, ne présentent pas les caractéristiques du lin. Une partie d'une lisière a été conservée, réalisée avec deux fils plus gros que ceux du tissu, d'un diamètre supérieur à $1 \mathrm{~mm}$. Cet exemple est très probablement un tissu utilitaire, fabriqué avec deux matériaux différents, démontrant ainsi le savoir-faire et la capacité d'adaptation des artisans textiles d'Akrotiri ${ }^{26}$.

\section{Archéologie expérimentale}

Plusieurs tentatives de reconstitution de motifs ou de costumes entiers présents sur les peintures murales d'Akrotiri ont été entreprises $^{27}$. Déjà, dans les années 1920, le Lycée des Femmes Helléniques d'Athènes avait effectué des reconstitutions de costumes d'Akrotiri sous la direction d'Anna Apostolidou, directrice du musée des Arts décoratifs (aujourd'hui musée hellénique d'Art folklorique à Athènes). Récemment, Abby Lillethun a reconstruit le corsage d'une jeune fille qui cueille le crocus et en a étudié la coupe, la matière et la similitude avec les 
peintures murales ${ }^{28}$. Bernice Jones a aussi travaillé sur la confection et la reconstitution de costumes d'Akrotiri et de Crète $^{29}$.

En 2009, une tentative de reconstitution d'une partie d'un tissu d'Akrotiri en employant les mêmes matériaux et outils a été entreprise par ARTEX ${ }^{30}$; le tissage s'est fait sur un métier vertical, construit sur le modèle des métiers de l'époque classique (fig. 6). Les pesons utilisés ont été fabriqués à l'image de pesons discoïdes découverts à Akrotiri et les fils sont similaires à ceux découverts sur les tissus les plus fins, à savoir des fils de lin de $0,36 \mathrm{~mm}$ de diamètre. Bien qu'on ait essayé de respecter les caractéristiques du tissu original, la même densité n’a pu être obtenue. Ceci pose la question de l'éventuel recours à de l'huile d'olive afin d'humidifier les fils et de produire des tissus plus denses. Cette pratique, connue à travers les sources écrites antiques ${ }^{31}$, est attestée en Grèce jusqu'au Xx ${ }^{\mathrm{e}}$ siècle.

\section{Conclusion}

La ville antique d'Akrotiri constitue donc une source exceptionnelle d'information sur l'activité textile à l'âge du Bronze dans le monde égéen. Les sources iconographiques fournies par les nombreuses peintures murales, la présence de pesons et, dans une moindre mesure, de fusaïoles, ainsi que les fragments de tissus que nous pouvons compter parmi les plus anciens témoignages issus de contextes domestiques offrent un panorama riche et complexe de la façon dont les habitants d'Akrotiri pratiquaient et utilisaient les textiles.

Concernant l'organisation et la division des activités textiles, notamment celle du filage et du tissage, la répartition des poids limitée à quelques zones spécifiques témoigne de la présence de véritables ateliers de tissage qui devaient alimenter les besoins locaux et vraisemblablement être au cœur d'échanges à moyenne et longue distance.

Les travaux d'identification des matières premières soulignent l'importance et la maîtrise du travail des matières végétales pour la réalisation non seulement de filets, cordes, tresses, vanneries, sparteries, mais également de tissus. Les premiers résultats attestent que la matière première était puisée dans l'environnement immédiat.

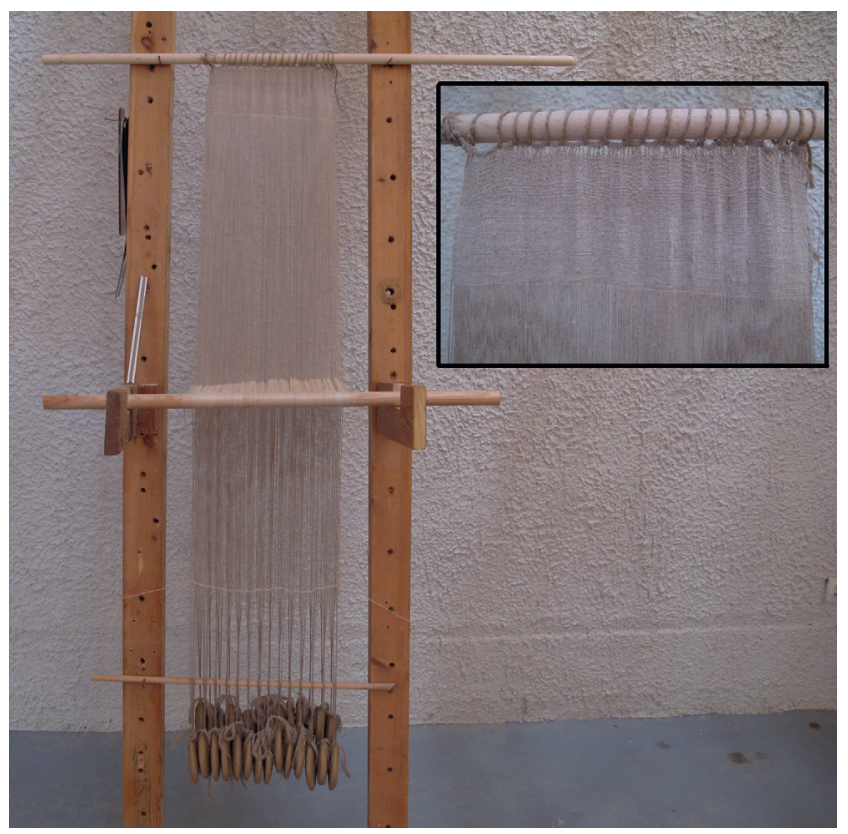

Fig. 6. Détail de la reconstitution du tissu du pilier 52n sur le métier vertical. (c) ARTEX/Stella Spantidaki.

D’autre part, les quelques fragments de tissus préservés et étudiés sur ce site ont permis de confirmer que la représentation des costumes sur les fresques s'appuyait vraisemblablement sur de véritables exemples et non sur la seule imagination de l'artiste. Ces éléments concernent pour l'essentiel les pièces de passementerie que l'on observe sur la plupart des représentations féminines.

Au sein de cet univers d'origine essentiellement végétale, un ensemble de fragments de tissus de laine confirme les informations délivrées par les tablettes en Linéaires A et B, à savoir l'importance de ce matériau dans la production textile. De même, la présence d'un cocon de soie sauvage suggère une éventuelle activité de sériciculture au cœur de la mer Égée et d'une façon générale en Méditerranée à l'âge du Bronze sans que l'on puisse cependant en imaginer l'ampleur.
Notes

1. Doumas, 1999.

2. Jones, 1998 ; Jones, 2003, p. 441-450 ; Lillethun, 2003, p. 463-471 ; Spantidaki, 2005 ; Nosch, 2008, p. 1-12.

3. Tzachili, 2007a, p. 193

4. Tzachili, 2007b, p. 262.

5. Tzachili, 1990, p. 381 ; idem, 2007a, p. 194 ; idem, 2007 b, p. 265.

6. Tzachili, 2007 b, p. 271.

7. Vakirtzi, thèse en cours.

8. Tzachili, 2007b, p. 259-261.

9. Vakirtzi, 2013, p. 234.

10. Vakirtzi, sous presse; Tzachili, 2007a, p. 192
11. Tzachili, 2007a, p. 192.

12. Mårtensson et al., 2009, p. 373-398; Andersson Strand, 2012, p. 207-214.

13. Tzachili et al., 2015.

14. Tzachili, 2007b, p. 277-280.

15. Au cours de la croissance de la plupart des plantes, des dépôts solides de silice se forment dans les espaces intracellulaires et extracellulaires. Ces dépôts de silice, ainsi que d'autres dépôts minéraux sont appelés «phytolithes».

Chacun à des morphologies caractéristiques, qui constituent souvent une empreinte de la cellule, ce qui leur confère une signification taxonomique. 32-38.

16. Spantidaki et Moulherat 2004

17. Idem, 2006, p. 284-289. fig. 4-6.

18. Spantidaki et Moulherat 2006, 287,

19. Ibid., p. 287, fig. 7 ; Doumas 1999 , p. 150 , fig. 114 .

20. Moulherat et Spantidaki 2008, p. 37-42.

21. Panagiotakopulu, 1997, p. 421.

22. Chrysikopoulou, 2005, p. 77-80.

23. Le Centre Hellénique de recherche et de restauration de tissus archéologiques a été fondé en 2002 par Iris Tzachili (archéologue), Youlie Spantidaki (biochimiste, spécialiste de tissus anciens) 
et Eliza Polychroniadou (restauratrice textile). Le Centre a collaboré avec Christophe Moulherat dès 2002 dans l'étude de tissus archéologiques grecs.

24. Moulherat et Spantidaki, 2007, p. 49,50 .

25. Spantidaki et Moulherat, 2012,

p. 188 , fig. 7.2 .

26. Ibid., fig. 7.1 .

27. Marcar, 2005, p. 30-43 ; Spantidaki, 2008, p. 43-48.

28. Lillethun, 2003, p. 463-471.

29. Jones, 2003, p. 441-450.

30. Spantidaki, 2009-2010, p. 75-84.

31. Hom. Od. 7.107 ; Hp. Salubr. 3.6.

\section{Références bibliographiques}

Andersson Strand E., 2012, "From spindle whorls and loom-weights to fabrics in the Bronze Age Aegean and Eastern Mediterranean", dans M.-L.Nosch, R. Laffineur (dir.) KOSMOS. Jewellery, Adornment and Textiles in the Aegean Bronze Age. Proceedings of the $13^{\text {th }}$ International Aegean Conference, Danish National Research Foundation's Centre for Textile Research, 21-26 April 2010. Aegeaum 33, p. 207-214.

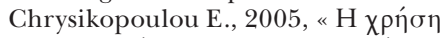

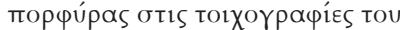

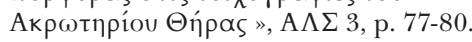

Doumas Chr., 1999 (1992), O1 torxorpapíes tпs $\Theta \eta ́ p \alpha s$, Thera Foundation.

Emery I., 1994, The primary structures of fabrics, Thames and Hudson, London.

Jones B., 1998, Minoan Women's Clothes. An investigation of their construction from the Depictions in Aegean Art, New York.

Jones B., 2003, "Veils and Mantles : An investigation of the construction and function of the costumes of the veiled dancer from Thera and the camp stool banqueter from Knossos", dans K. P. Foster, R. Laffineur (dir.) Metron. Measuring the Aegean Bronze Age. Proceedings of the $9^{\text {th }}$ International Aegean Conference, New Have, Yale University, 18-21 april, 2002, Aegeaum 24, p. 441-450, Pl. LXXXIV-XC.

Lillethun A., 2003, "The recreation of Aegean cloth and clothing", dans K. P. Foster, R. Laffineur (dir.) Metron. Measuring the Aegean Bronze Age. Proceedings of the $9^{\text {th }}$ International Aegean Conference, New Have, Yale University, 18-21 april, 2002, Aegeaum 24, p. $463-471$.

Marcar A., 2005, "Reconstructing Aegean Bronze Age Fashions", dans L. Cleland, M. Harlow, L. Llewellyn-Jones (dir.) The Clothed Body in the ancient World, Oxbow Books, p. 30-43.

Mårtensson L., Nosch M.-L., Andersson Strand E., 2009, "Shape of Things: Understanding a Loom-wheight", Oxford Journal of Archaeology (28) 4, p. 373-398.

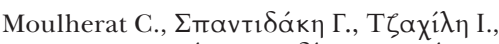

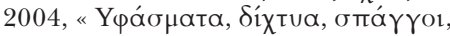

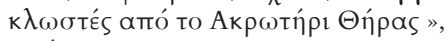
Apáxvi 2, p. 15-19.

Moulherat C., Spantidaki Y., 2007, "Preliminary results from the textiles discovered in Santorini", dans A. RastEicher, R. Windler (dir.) Archäologische Textilfunde. NESAT IX, Nordeuropäisches Symposium für archäologische Textilien. Braunwald, 18-20 Mai 2005, Ennenda, p. 49-52.

Moulhérat C., Spantidaki Y., 2008,

"Première attestation de laine sur le site protohistorique d'Akrotiri à Théra ", dans C. Alfaro, L. Karali (dir.) Purpureae Vestes II. Textiles and Dyes in Antiquity. Proceedings of the $2^{\text {nd }}$ International Symposium on Textiles and Dyes in Antiquity, Athens, 24-26 November, 2005, University of Valencia, p. 37-42.

Nosch M.-L., 2008, "Haute Couture in the Bronze Age: A History of Minoan Female Costumes from Thera", dans M. Gleba, C. Munkholt, M.-L. Nosch (dir.) Dressing the Past, Oxbow Books, Ancient Textile Series 3, p. 1-12.

Panagiotakopulu E., 1997, "A lepidopterus Cocoon from Thera and Evidence for Silk in the Aegean Bronze Age", Antiquity 71, p. 420-429.

Seiler-Baldinger A.M., 1994, Textiles. A classification of techniques, Crawford House Publishing.

Spantidaki S., 2008, "Preliminary results of the reconstruction of Theran textiles", dans C. Alfaro, L. Karali (dir.) Purpureae Vestes II. Textiles and Dyes in Antiquity.Proceedings of the $2^{\text {nd }}$ International Symposium on Textiles and Dyes in Antiquity, Athens, 24-26 November, 2005, University of Valencia, p. 43-48.

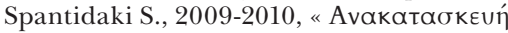

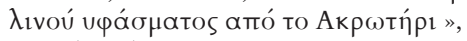
$\mathrm{A} \Lambda \Sigma 7$, p. $75-84$.

Spantidaki Y., Moulherat C., 2004,

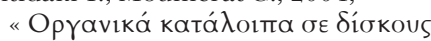
కurapiás ", $A \Lambda \Sigma$ 2, p. 32-38.

Spantidaki Y., Moulherat C., 2006,

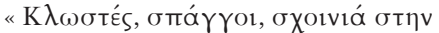

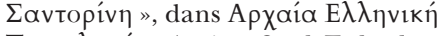
TExvoגoría. Ancient Greek Technology, Proceedings of the $2^{\text {nd }}$ International

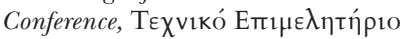

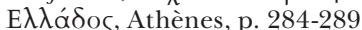

Spantidaki Y., Moulherat C., 2012, "Greece", dans M. Gleba, U. Mannering (dir.) Textiles and Textile Production in Europe: From Prehistory to $A D$ 400, Oxbow Books, Ancient Textile Series 11, p. 185-200.

Tzachili I., 1990, "All important yet elusive: Looking for Evidence of Cloth-making at Akrotiri", dans D. A. Hardy (dir.) Thera and the Aegean World III, vol. 1 . Proceedings of the Third International Congress, Santorini, Greece, 3-9 September 1989, Londres, The Thera Foundation, p. $380-389$.
Tzachili I., 1997, Yqavtıkí kaı Yфávtpes бто Проїбторıко́ Aıraío. 2000-1000

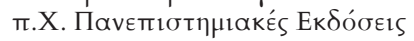

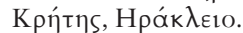

Tzachili I., 2007a, "Weaving at Acrotiri, Thera: Defining Cloth-Making Activities as Social Process in a Late Bronze Age Aegean Town", dans C. Gillis, M.-L. Nosch (dir.) Ancient Textiles. Production, Craft and Society. Proceedings of the First International Conference on Ancient Textiles, held at Lund, Sweden, and Copenhagen, Denmark, on March 19-23, 2003, Oxbow Books, Ancient Textile Series 1, p. 190-196.

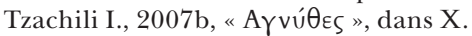

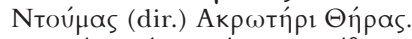

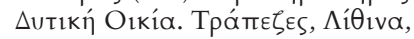
Mєta $\lambda \lambda_{1 \kappa} \alpha$, Поюкí $\lambda \alpha$, Athènes, p. 262-271.

Tzachili I., Spantidaki S., Andersson Strand E., Nosch M.-L., Cutler J., 2015, “Textile Tools from Acrotiri, Thera, Greece", dans E. Andersson Strand, M.-L. Nosch (dir.), Tools, Textiles and Contexts: Textile Production in the Aegean and Eastern Mediterranean Bronze Age, Oxbow Books, Ancient Textile Series. Sous presse.

Vakirtzi S., 2013, "The decorated spindlewhorls from prehistoric Akrotiri, Thera", dans A. Papadopoulos (dir.), TA $\Lambda$ ANTA. Proceedings of the Dutch archaeological and historical society, Vol. XLIV (2012), p. 227-244.

\section{Documents inédits}

Spantidaki S., 2005, L'archéologie des tissus en Grèce ancienne. Le cas de Santorin. Mémoire de D.E.A., Université de Paris Sorbonne (Paris IV).

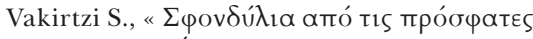

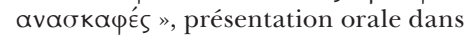
la journée d'études à l'occasion des 40 ans des fouilles à Akrotiri, $\mathrm{H} \varepsilon v$

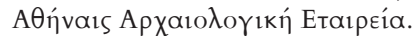

Vakirtzi S., Le filage aux îles égéennes de l'âge du Bronze à travers la présence de fusaïoles aux sites archéologiques : étude des formes, de la fonctionnalité et de la dispersion des accessoires du fuseau découverts dans des habitats et des cimetières, Thèse en cours, Université de Crète.

\section{Sources antiques}

Hom. Od. : Homère, Odyssée, Tome I, Chants I-VII. Texte établi et traduit par V. Bérard, CUF, 2002 (1924).

Hpc. Salubr. : Littré É., Euvres complètes d'Hippocrate, vol. VI, Amsterdam, Hakkert, 1962 (1849). 